Revista Signos 2009, 42(70) 245-269

\title{
Metáforas y argumentación: Lugar y función de las metáforas conceptuales en la actividad argumentativa
}

\author{
Cristián Santibáñez \\ Universidad Diego Portales \\ Chile
}

Resumen: El artículo sugiere una forma de entender y analizar el funcionamiento de las metáforas conceptuales desde la perspectiva de la práctica argumentativa. Para el efecto, se discuten tanto algunos de los principales aportes de la lingüística cognitiva en el análisis de las metáforas, como la perspectiva de Stephen Toulmin (1958) para la reconstrucción de argumentos conocida como lógica factual. El paralelo entre el diseño de descripción metafórica y el modelo de la lógica factual, arroja que las metáforas conceptuales operan como 'apoyos' argumentativos cuando se manifiestan como asunciones sociales, campo de experiencia o evidencia general, y como 'garantías' cuando una de las correspondencias se manifiesta como ley de paso entre las premisas y la conclusión del argumento.

Palabras Clave: Metáforas conceptuales, argumentación, apoyo, lógica factual, proverbios.

Recibido: 26-II-2008 Aceptado: 30-XII-2008
Correspondencia: Cristián Santibáñez (cristian.santibañez@udp.cl). Universidad Diego Portales. Avenida Ejército Libertador 278, Santiago, Chile. 


\title{
Metaphors and argumentation: Place and function of conceptual metaphors in argumentative activity
}

\begin{abstract}
This paper suggests a way to understand and analyze the working of metaphors from the point of view of argumentative practice. For this purpose, it discusses not only the main contributions made by cognitive linguistics to the analysis of metaphors, but also Stephen Toulmin's (1958) perspective for argumentative reconstruction, known as factual logic. The parallel between the metaphorical descriptive design and working logic model shows that the conceptual metaphors work as 'backing' when they are manifested as social assumptions, experience or general evidence, as well as 'warrants' when one of the correspondences is manifested as a passage-law between the premises and the conclusion of the argument.
\end{abstract}

Key Words: Conceptual metaphors, argumentation, backing, factual logic, proverbs.

\section{INTRODUCCIÓN}

Ejemplos de usos estratégicos de las palabras se encuentran todos los días. En Atenas, para dar un caso, existe una compañía de mudanzas que se llama mehaphoró. Es una buena estrategia, un uso inteligente de una metonimia -el nombre por la función-, para sustituir y reducir la función de transferir, que la compañía realiza a diario, a través del nombre de la misma. Pero, tal como el nombre de la compañía solo representa la función general de transferir muebles y objetos de un lugar a otro, así también el acercamiento retórico estándar al término 'metáfora' solo describe y reduce este tropo como "changing a word from its literal meaning to one not properly applicable but analogous to it" (Lanham, 1991: 188).

Esta definición sigue una venerable tradición que probablemente comenzó con Aristóteles. En Retórica, hablando sobre estilo, Aristóteles (2001: 272) concluye que:

"Sea nuestro punto de partida el siguiente: que aprender con facilidad es algo naturalmente agradable para todos y que, por otra parte, las palabras tienen un significado determinado, así que los nombres que nos enseñan algo son los más agradables. En consecuencia, las palabras raras nos son desconocidas; las precisas ya las conocemos, así que es la metáfora la que consigue mejor lo que buscamos. En efecto, cuando el poeta llama a la vejez "rastrojo" produce en nosotros un aprendizaje y el conocimiento a través de una clase, pues ambas cosas implican que algo se ha marchitado".

Luego el Estagirita añade: "Así que la mayoría de las expresiones ingeniosas se consigue por medio de la metáfora y por haber conseguido previamente engatusar al oyente" (Aristóteles, 2001: 281). Estos comentarios condenaron a la metáfora a ser, por un largo periodo, solo materia de la imaginación y la poesía o, en el mejor de los casos, un 'divertimento' estratégico. Al mismo tiempo, Aristóteles reconoce en estas notas que la metáfora es un recurso natural en el discurso, siempre a la mano para ser usado. 
En el análisis del discurso y la comunicación real, desde un punto de vista argumentativo, esta perspectiva ha sido utilizada por Perelman y Olbrechts-Tyteca (2000), pero después de ellos no mucho ha sido incorporado. En efecto, ni la Pragma-dialéctica (van Eemeren \& Grootendorst, 1992, 2004; van Eemeren \& Houtlosser, 2002a, 2002b, 2002c, 2003, 2008) ni otros esfuerzos teóricos en el campo de estudio de la argumentación (Ducrot \& Anscombre, 1983; Tindale, 1999; Walton, 1992, 2004, 2006, 2007) han posibilitado nuevos acercamientos al problema de la metáfora. Es una desatención que se observa con claridad toda vez que estas teorías de la argumentación han tratado de insertar los conceptos y figuras retóricas más importantes en el marco del análisis del fenómeno argumentativo. Esta desatención se convierte, por lo tanto, en una motivación, puesto que ya hace aproximadamente treinta años que ha emergido un importante punto de vista sobre este funcionamiento natural del lenguaje. Esta nueva aproximación sigue los avances que en las ciencias cognitivas, en especial en la lingüística, han tenido lugar.

Esta perspectiva sostiene que ciertos discursos están estructurados sobre la base de 'marcos' o 'sistemas conceptuales', y que tales 'marcos' son resultado de las metáforas. Esta posición ha sido, en particular, propuesta y defendida por Lakoff y su equipo para mostrar cómo la gente percibe, piensa y actúa en el mundo a través del lenguaje natural (Lakoff, 2006a, 2006b, 1994; Lakoff \& Turner, 1989; Lakoff \& Johnson, 1980; Kövecses, 2002). Desde el punto de vista de las ciencias cognitivas aplicadas, y después de un largo periodo de investigación empírica, tanto la lingüística como la psicología han sugerido que las similitudes en las concepciones de mundo entre diferentes culturas han sido producidas por el uso de metáforas comunes y compartidas. Una de las ideas centrales es que los valores fundamentales y las opiniones de una cultura particular son sistemáticamente coherentes con su estructura metafórica y marcos léxicos.

Un ejemplo recurrente en la bibliografía cognitivista, que ilustra este último punto, es lo que sucede en la conceptualización, generalizada en occidente, de las palabras 'sociedad' y 'nación' que envuelven categorías como 'persona' y 'familia', tal como se observa en las siguientes expresiones: "naciones vecinas", "los padres fundadores de la patria". Algo similar ocurre en la conceptualización de 'poder político', que se concibe como una 'fuerza física': "Sacaron a la oposición del gobierno". Los ejemplos son más claros cuando hablamos de 'amor', 'discusión' y 'teorías' en términos de 'viaje', 'guerra' y 'edificios', respectivamente (Lakoff \& Johnson, 1980).

A pesar de que ciertos aspectos o puntos de partida de este acercamiento pueden ser materia de discusión, tal como lo manifiesta la sustancial crítica de Davidson (1978) a nivel filosófico, es razonable considerar algunos aspectos de esta propuesta para ejercitar nuevas formas de descripción y explicación de la metáfora y su papel en la elaboración de argumentos.

De este modo, el presente trabajo tiene por objetivo fundamental entregar una propuesta teórica y metodológica que vincule, por un lado, los avances en los estudios de la metáfora desde 
un punto de vista cognitivo y, por otro lado, las reflexiones y teorías existentes en el campo de los estudios de la argumentación. Específicamente, se vinculará el análisis impulsado por Lakoff y Johnson (1980) y Lakoff y Turner (1989) en el ámbito de la metáfora, y el modelo de análisis de argumentos propuesto por Toulmin (1958) y Toulmin, Rieke y Janik (1979), para observar qué papel le cabría a la metáfora conceptual en el engranaje argumentativo.

Para este propósito, en la primera sección se incluyen dos apartados en los que se sintetizan, primero, los principales conceptos provenientes de la lingüística cognitiva en el análisis de metáforas conceptuales y, luego, una descripción del modelo de Toulmin (1958) con el objeto de explicar su modo de funcionamiento en la reconstrucción argumentativa. En la segunda sección, se entrega una metodología para el análisis de expresiones metafóricas. En la tercera sección de este trabajo se presenta el análisis de los casos reales bajo observación, que fueron escogidos tanto por su claridad como por representar de buena forma el uso de metáforas conceptuales con fines argumentativos. Las conclusiones exponen puntos críticos que pueden pasar a formar parte de futuras investigaciones que se preocupen por el lugar y función de las metáforas conceptuales en la actividad argumentativa.

\section{Marco de referencia}

\subsection{Teoría cognitivista de la metáfora}

Expresiones como las siguientes: "No creo que esta relación vaya a alguna parte", o "Mira lo lejos que hemos llegado", muestran que una 'relación personal', que podría incluso contener 'amor', es entendida como un 'viaje', siendo este último concepto el que exporta su significado, o su serie de connotaciones, al primero.

Una explicación resumida de este fenómeno, desde un punto de vista lingüístico cognitivo, sostiene que el entendimiento humano está compuesto por un set de correspondencias entre un dominio fuente y un dominio meta. En el ejemplo, el dominio fuente es VIAJE (A) y el dominio meta es RELACIÓN PERSONAL o AMOR (B). Elementos conceptuales constituyentes de B corresponden a elementos constituyentes de A. El proceso de correspondencias entre los dos dominios es llamado 'mapeo' en la terminología de Lakoff y Johnson (1980). De este modo, una expresión cotidiana es manifestación de una metáfora conceptual que involucra dos dominios conceptuales, en los que un dominio es conceptualizado en términos del otro.

Es importante añadir que, para Lakoff y Johnson (1980), el punto de partida es un nivel filosófico que ellos llaman 'experiencialista', que implica que los dominios fuente son dimensiones más cercanas o inmediatas a la experiencia corporal y que los dominios meta son más abstractos, lo que redunda en que, en general, el último importa significado desde el primero. Porque las 
metáforas conceptuales provienen de nuestra interacción con y en la experiencia, de la cual son producto, tal como las metáforas de orientación -FELIZ ES ARRIBA, TRISTE ES ABAJO-, ellas se manifiestan lingüísticamente. Las metáforas lingüísticas en el nivel de las expresiones son manifestaciones de metáforas conceptuales en el nivel del entendimiento, el pensamiento y los conceptos. Las metáforas conceptuales exponen mapeos sistemáticos entre dominios, pero al mismo tiempo son selectivas, esto es, enfatizan ciertos aspectos de la experiencia y esconden otros. Kövecses (2002:76) explica el marco general de esta situación con precisión:

"On what basis do we select the source domains for particular targets? In the traditional view, the selection of sources assumes an objective, literal and preexisting similarity between the source and the target. By contrast, the cognitive view maintains that the selection of source domains depends on human factors that reflect nonobjective, nonliteral, and nonpreexisting similarities between a source and a target domain. These are called the experiential bases or motivation of conceptual metaphors. Some of the common kinds of such similarities include: (1) correlations in experience, (2) perceived structural similarity, (3) perceived structural similarity by basic metaphors, (4) source being the root of the target”.

Los investigadores en esta línea han desarrollado muchos conceptos para explorar todo lo que las metáforas significan, batería de términos que no es posible detallar aquí. Sin embargo, una mención especial cabe hacer respecto del concepto 'metáforas convencionalizadas' (Lakoff \& Johnson, 1980; Kövecses, 2002), y respecto del término 'imagen-esquema' (Lakoff \& Turner, 1989), porque ambos permitirán desarrollar parte del análisis al corpus seleccionado.

Por 'metáforas convencionalizadas' Lakoff y Johnson (1980) entienden aquel tipo de metáfora conceptual que es parte de la manera natural de un grupo o comunidad de pensar y entender un dominio conceptual. A diferencia de la noción de 'convención' en filosofía y lingüística tradicional ligada a la idea de 'arbitrariedad', la noción de 'convención' en estos lingüistas cognitivos se vincula con un tipo de construcción con raigambre comunitario, es decir, un tipo de formación semántica con una larga historia de uso. Lakoff y Johnson (1980) señalan que hay 'grados de convencionalidad' en las metáforas conceptuales, en el sentido de que hay algunas de ellas que están más convencionalizadas que otras, como por ejemplo AMOR ES VIAJE, frente a AMOR ES UNA OBRA DE ARTE COLABORATIVA que podría ser una metáfora conceptual con una corta vida en una comunidad, e incluso, solo ser parte de un sub-grupo de esa comunidad, como es el caso de las metáforas utilizadas por escritores y poetas. Por su parte, con el concepto de 'esquemaimagen', Lakoff y Turner (1989) se refieren a la existencia de patrones o esqueletos generales de inferencia metafórica que exportan información general de un dominio sobre otro, es decir, ciertas características de un dominio fuente que orientan el proceso de entendimiento de un dominio meta. 
Son precisamente estos dos últimos conceptos los que permiten plantear una relación ya más específica entre el análisis de metáforas conceptuales y el análisis de argumentos, ya que ambos muestran que en el habla metafórica hay un proceso básico de inferencia entre dominios. Pero aun persisten algunos problemas sustanciales que se pueden resumir, al menos, en dos preguntas: ¿cuál es la función o lugar de una metáfora conceptual en un argumento real?, y en particular, ¿cómo podría integrarse esta perspectiva a una teoría de la argumentación?

Algunas respuestas preliminares han sido dadas por Lakoff $(2004,2006 a)$, a pesar de que él no es un experto en teoría de la argumentación o retórica, pero sus intuiciones son plausibles cuando analiza, por ejemplo, argumentos políticos. Comentando la estrategia argumentativa y retórica del Partido Republicano de Estados Unidos, enfatiza la idea de que los integrantes de este partido definen los temas de la agenda nacional norteamericana según sus marcos conceptuales, y que estos circunscriben tanto los problemas a los que se dirigen como las soluciones que plantean. Sintetiza su perspectiva como sigue:

"They have moral premises, that is, they are about what is right; they use versions of contested values taken from a particular moral view; they have an implicit or explicit narrative structure, i. e., they all tell stories with heroes, villains, victims, common themes, etc.; they also serve as counterarguments: they undermine arguments on the other side; they have issue-defining frames that set up problem and the solution; they use commonplaces frames -frames known so widely that they resonate immediately, whether true or not; they use language with surface frames that evoke deeper frames" (Lakoff , 2006a: 119).

Para Lakoff (2006a) los marcos de superficie, que evocan marcos más profundos en el entendimiento del mundo, se obtienen de las expresiones metafóricas. Con mayor claridad, Lakoff ilustra esto proponiendo el análisis del uso del Bad apple frame (el marco de la manzana podri$d a$ ), contenido en el dicho popular "una manzana podrida echa a perder el cajón", marco que funciona en diferentes culturas en tanto lugar común:

"Bad apple frame: Consider the saying "A bad apple spoils the barrel". The implication is that if you remove the bad apple or some small number of bad apples, the others will be fine. The rot is localized and will not spread. Rot here is a metaphor for immorality. In a case where there is immoral behavior, it points blame at one person or a few people -and not to any broader systematic immorality, an immoral policy, or an immoral culture. This commonplace frame has been used to limit the inquiry into torture as a systematic problem in the military (as in the Abu Gharaib scandal), so the problem is contained. The army just got rid of the "bad apple" -the lowest-ranking military personnel involved. The same was true of Enron Corporation, where a few executives (Jeffrey Skilling and Kenneth Lay) were identified as bad apples, rather than the entire culture of Enron, where top-level and even midlevel employees commonly schemed to rip off the public by taking advantage 
of the deregulation of utilities with illegal actions like those code-named "Death Star" and "Get Shorty" (Lakoff, 2006a: 126).

Más allá del sugestivo análisis de contenido expuesto en la cita anterior, aun la perspectiva del marco conceptual aparece difusa desde una perspectiva teórica de la argumentación, porque técnicamente no se obtiene inmediatamente cuál es el lugar y función que la metáfora conceptual puede tener en la cadena silogística y/o entimemática. Porque las metáforas funcionan con consistencia cultural, a través de procedimientos mentales, sugiero que la metáfora conceptual se considere como 'apoyo' o como 'garantía' en términos del modelo de Toulmin (1958), de acuerdo a la discusión y criterios que a continuación se ensayan.

\subsection{El modelo de argumentación de Toulmin}

En 1958 aparece en Inglaterra el libro The uses of argument de Stephen Toulmin. El objetivo de este libro era posicionar una crítica epistemológica que trataba de cuestionar tanto el modo tradicional de concebir la producción del conocimiento, como el hábito científico de considerar como fundamento del razonar, incluso para la vida cotidiana, el proceso silogístico aristotélico.

A partir del título en inglés del tercer capítulo de su libro, a saber, Working logic, se ha difundido su propuesta en español bajo el nombre de 'lógica factual'. Como así lo indica su nombre, el interés de la propuesta está en analizar el modo en que el razonamiento se desenvuelve en contextos específicos. Para el efecto, Toulmin (1958) ocupa el proceso general en el que la jurisprudencia se despliega, esto es, sopesando la fuerza de las leyes de acuerdo a los casos y criterios de aplicación. Fuerza y criterio son los dos términos matrices a balancear para obtener una descripción genuina de la exposición de argumentos. Este balance lo logra Toulmin construyendo un modelo con seis entradas, superando así la lógica aristotélica de tres (premisa mayor, premisa menor y conclusión).

Las entradas del modelo, que deviene en método para la confección y análisis de argumentos, son:

1. Apoyo: cuerpo de contenidos desde donde emanan las garantías y que nos remite al mundo sustancial en el que encontramos investigaciones, textos, códigos, supuestos sociales que nos permiten afirmar una garantía.

2. Garantía: principio general, norma tácita, supuesto o enunciados generales, de naturaleza formal, que permiten el paso de los datos a las conclusiones.

3. Datos: son de orden empírico o factual, y permiten la emergencia de una pretensión o conclusión. 
4. Conclusión: son las pretensiones, demandas o alegatos que buscan, entre muchos de sus posibles propósitos, posicionar una acción, una perspectiva.

5. Cualificadores modales: son construcciones lingüísticas que permiten cambiar la fuerza pragmática de una pretensión.

6. Condiciones de refutación: son excepciones que debilitan a la garantía, son parte de la información contenida en los apoyos, y socavan la fuerza final de la conclusión.

El modelo se lee de la siguiente forma: Dada nuestra experiencia general del campo en cuestión (apoyo), y de acuerdo con las reglas o principios resultantes de tal experiencia (garantía), utilizando los siguientes hechos específicos (datos), de una forma cualificada (cualificador modal), se permite concluir lo siguiente (conclusión o pretensión), a menos que exista una específica condición de refutación (excepciones). Como se desprende, el nombre de 'lógica factual' proviene del hecho de que el modelo está siempre en relación con los acontecimientos, tanto a través de los 'apoyos', como de los datos y las excepciones, siendo el caso que la 'conclusión' y los 'cualficadores modales' también nos vinculan a las estrategias que los hablantes tienen para instalar sus posiciones en debates y controversias. Un ejemplo para ilustrar la aplicación de este modelo, que se obtiene de Toulmin et al. (1979: 87) es el siguiente:

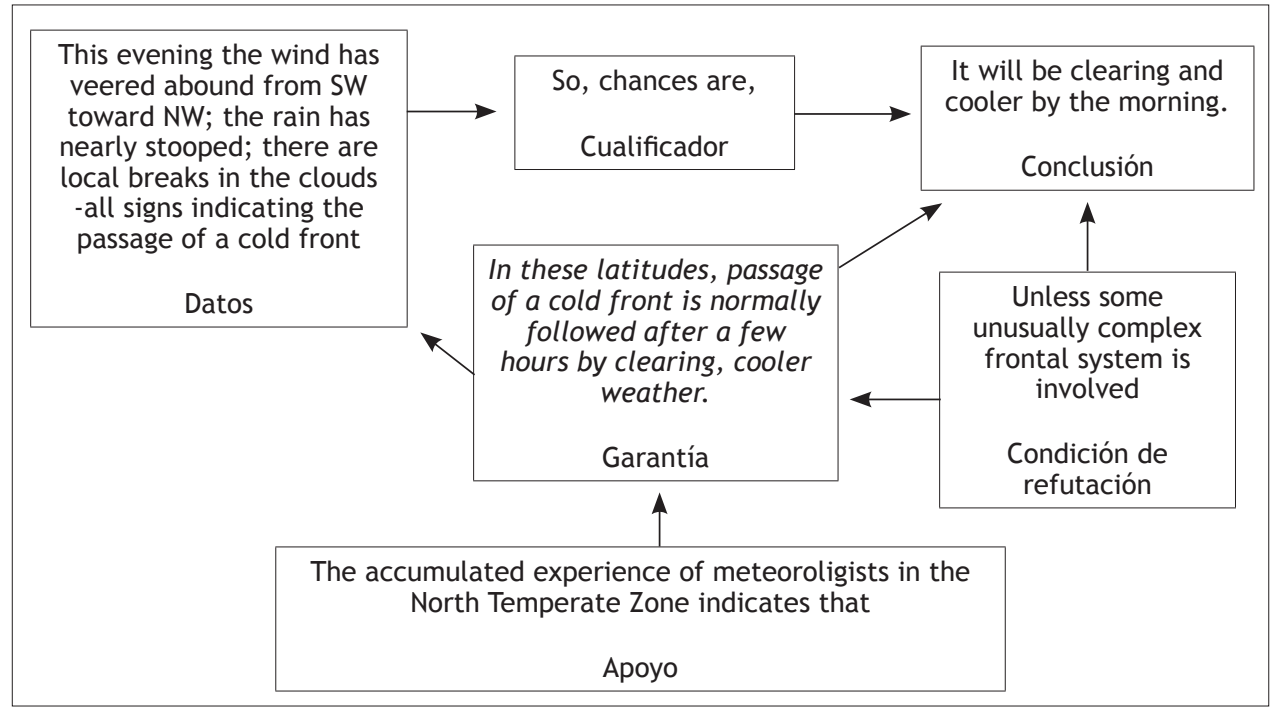

Figura 1. Ejemplo de la estructura visual del modelo de Toulmin (1958). 
Cada una de las categorías del modelo se ha aplicado conforme a la razonabilidad del campo específico de argumentación que, en el ejemplo anterior, se refiere al campo climático. El modelo muestra la fuerza que adquiere cada una de las partes y, sobre todo, el carácter esencial que tiene la categoría 'apoyo' para sentar la base de inferencia sobre la que descansa el argumento en su totalidad. De acuerdo a su definición, el 'apoyo' es el conjunto general de supuestos con profundas raíces en el cuerpo social. Este conjunto de supuestos a veces se encuentra fuertemente institucionalizado -como por ejemplo en el campo jurídico a través de los códigos-, o menos institucionalizados pero formando parte de una costumbre arraigada -como por ejemplo en el campo fraseológico a través de dichos y proverbios. En ambos casos, los apoyos van formando parte, generación tras generación, de los contenidos tácitos que estructuran dominios y comunidades discursivas, tal como Lakoff y Johnson (1980) postulan el alcance de las metáforas conceptuales. Este paralelo teórico es el que se pondrá a prueba, a continuación, analizando casos reales obtenidos de distintos ámbitos sociales.

\section{Marco metodológico para el análisis de metáforas conceptuales}

Antes de ir a lo anunciado, se debe realizar una descripción metodológica para distinguir el proceso por el cual el mapeo entre dominios puede ser analizado, esto es, una metodología que permita el análisis de metáforas conceptuales y, a su vez, distinguir la proyección que cabría desde el análisis metafórico al análisis argumentativo.

Este dispositivo metodológico (Kövecses \& Szabó, 1996; Kövecses, 2002) se compone de seis pasos: 1) obtención de expresiones, 2) distinción de los dominios conceptuales, 3) nombre de la metáfora conceptual contenida en las expresiones, 4) caracterización aspectual del dominio fuente, 5) descripción de la lógica situacional del dominio fuente, y 6) disposición de correspondencias o mapeo entre los dominios.

Un ejemplo que permite observar la aplicación de este diseño, ejemplo también recurrente en la literatura cognitivista (Lakoff \& Johnson, 1989; Kövecses, 2002), se obtiene, precisamente, siguiendo los pasos de la metodología expuesta:

1. Expresiones metafóricas: "Tu posición es indefendible", "Atacó cada uno de los puntos débiles de tu argumento", "Sus críticas dieron justo en el blanco", "Nunca he ganado una discusión con él", "Me mató con su alegato", "Su táctica en el debate era efectiva".

2. La mera intuición ya orienta tanto los conceptos metafóricos que están implicados como la relación básica que existe entre ellos. Los conceptos, evidentemente, son: 'discusión' y 'guerra'. 
3. Así, se podría sostener que la metáfora conceptual lleva por nombre: LAS DISCUSIONES SON GUERRAS, donde el dominio fuente es 'guerra' y el dominio meta es 'discusión'.

4. La caracterización aspectual del dominio fuente rastrea las propiedades básicas que éste tiene, lo que a su vez permite dirigir el sentido de la lógica situacional; en este ejemplo, algunas propiedades básicas que cabría instalar son las siguientes: 'las guerras requieren tiempo', que varía de acuerdo con las estrategias militares aplicadas; 'las guerras suponen gasto de energía física, mental, económica y social'; 'las guerras producen víctimas y victimarios'; 'las guerras se producen, entre otras razones, por diferencias irreconciliables, intereses específicos para lograr ventajas, o abusos de algún tipo (posición, fuerza, estatus, etc.)'.

5. La lógica situacional del dominio fuente, en virtud de las propiedades y la concretísima experiencia que tenemos en él, demuestra entre otras cosas que: 'a mayor tiempo atacando, mayor destrucción'; 'mayor poder de las armas usadas en el ataque, mayor debilitamiento del lugar atacado'; 'mejor estrategia de ataque y defensa, mejor el resultado en el tiempo'; 'mayor precisión en el ataque, más rápidamente se obtiene la victoria'.

6. En este esquema el último paso, quizá el más importante, es la disposición efectiva de las correspondencias entre los dominios comprometidos en la metáfora conceptual, que para el caso serían: 'tal como en la guerra el mejor resultado se obtiene en función de la mejor táctica militar, así una discusión se gana en función de la mejor estrategia retórica y argumentativa'; 'así como en una guerra la victoria se consigue atacando los flancos más débiles del enemigo, así la victoria en una discusión se alcanza atacando los puntos más débiles de los argumentos del oponente'.

\section{Análisis de casos metafóricos}

Los casos que a continuación se exponen fueron seleccionados por su claridad y por representar tanto el discurso político chileno, como del uso de proverbios en las discusiones científicas contemporáneas. Serán analizados, primero, exponiendo el extracto de discurso del que provienen; segundo, distinguiendo expresiones metafóricas y correspondencias; y tercero, aplicando un paralelo entre el modelo de análisis cognitivo de metáforas y el esquema visual del modelo de Toulmin (1958) para el análisis de argumentos, con el objeto de observar la función que les cabría a las metáforas conceptuales en la cadena argumentativa.

\subsection{Metáforas en la vida económica}

Como todos sabemos, compartimos muchas metáforas en la actual economía global. Por ejemplo, una muy común entre los americanos es la que se obtiene de la expresión 'tax relief' -que 
suele traducirse como 'alivio impositivo' o, en su defecto, 'rebaja de impuestos' en el ámbito local chileno-, metáfora que ha sido analizada en detalle por Lakoff (2004: 3-34). Esta es una expresión metafórica que también puede ser escuchada en Argentina, Francia, Inglaterra, Seúl, etc., donde sea que el sistema neoliberal esté funcionando. Otra metáfora conceptual es LOS SISTEMAS ECONÓMICOS SON EDIFICIOS, que se manifiesta en expresiones como: "con su economía en ruinas, el país no puede solventar un proyecto como ese".

Un muy buen ejemplo para mostrar la relación entre metáfora conceptual y argumentación en el campo de la economía, es el que a continuación se reproduce, obtenido del periódico electrónico chileno La Segunda de su edición del día 10 de octubre de 2008, que corresponde a la columna de opinión del economista Juan Andrés Fontaine:

\section{Juan Andrés Fontaine}

\section{Cómo capear el temporal}

Alerta roja en los mercados. El estruendoso derrumbe de Wall Street y otras plazas bursátiles no podía sino repercutir en Chile. Pero la virulenta alza del dólar, la caída de las bolsas y la elevación del costo del crédito nos han tomado por sorpresa. ¿Será que se nos viene el huracán? Un análisis frío de las condiciones actuales de la economía nacional debería despejar los temores, pero en tiempos de tormenta hasta las mejores naves pueden naufragar si no son piloteadas con destreza.

Los desaciertos cometidos por la dupla Paulson-Bernanke en el puente de mando de la economía americana han llevado a que un problema bancario peligroso, pero confinado a un segmento del crédito, pueda devenir en un pánico financiero global. Afortunadamente, las medidas necesarias para contener y subsanar la epidemia de desconfianza son abundantemente conocidas y de a poco han comenzado a ser aplicadas en Estados Unidos y Europa. Suponiendo que ellas surten efecto, el Fondo Monetario Internacional vaticina que el crecimiento mundial se desacelera al $3 \%$ el próximo año, desde el $4 \%$ previsto para el actual y el $5 \%$ estimado para el anterior. Aunque se trataría de un escenario negativo para Chile, los hemos conocido peores y no hace mucho. El crecimiento económico mundial fue de poco más de $2 \%$ en 2001, 1998 y 1991, alcanzando tan sólo $0,6 \%$ en el fatídico 1982. Sólo en dos de esas ocasiones, en 1982 y 1998, Chile en verdad se vio en apuros.

Los ahorros fiscales acumulados y las reservas internacionales totalizarán más de US\$ 50.000 millones hacia fines de año: impresionante suma, aunque semejante a la que, en relación ya sea a las importaciones o a la deuda externa de corto plazo teníamos en 1997, en la antesala de la crisis asiática. Las autoridades de entonces se ufanaban del grosor del blindaje y, bien sabemos, éste no fue suficiente para prevenir el naufragio económico de 1998-99. 
Un primer comentario que se puede hacer aquí es que, en general, la metáfora conceptual es un procedimiento para inferir verdades particulares desde principios y experiencias aceptadas en ambientes culturales específicos, lo que es, a su vez, una idea muy cercana al concepto de 'apoyo' en Toulmin (1958) y Toulmin et al. (1979). Por otra parte, no debe perderse de vista que en la perspectiva de Lakoff (Lakoff \& Johnson, 1980; Lakoff, 1994) la verdad es experiencial, materia de interacción, tal como el modelo jurisprudencial de argumentos de Toulmin (1958) sostiene respecto de que las conclusiones de un argumento están siempre sujetas a camposdependientes, de acuerdo a criterios de razonabilidad contextual.

En lo contenido en este caso, en el marco de un contexto completamente argumentativo como es el de una columna de opinión, es posible ver el funcionamiento de, al menos, dos metáforas conceptuales: LA ECONOMÍA ES UN ESTADO CLIMÁTICO y LA ECONOMÍA ES UNA NAVE, que se manifiestan, descontando el propio título que ya es indicativo de la metáfora de base, en expresiones como "Alerta roja en los mercados", y en especial en los siguientes dichos de su autor: "¿Será que se nos viene el huracán? Un análisis frío de las condiciones actuales de la economía nacional debería despejar los temores, pero en tiempos de tormenta hasta las mejores naves pueden naufragar si no son piloteadas con destreza."

Para la primera metáfora conceptual, las siguientes correspondencias entre los dominios involucrados pueden ser mencionadas: así como el estado del tiempo climático puede empeorar rápidamente, así una economía nacional puede enfrentarse rápidamente a cambios desfavorables; así como se debe estar preparado para peores cambios del estado climático, así también una economía debe adoptar medidas con rapidez para peores escenarios. Para la segunda metáfora conceptual cabe mencionar dos correspondencias que se relacionan con el punto de vista que se defiende en la columna de opinión: así como una nave en alta mar turbulento se salva de naufragar por la pericia de su capitán, así también una economía nacional en un contexto financiero internacional turbulento se salva de entrar en crisis por la destreza de su máximo responsable; así como el capitán de una nave en alta mar turbulento debe tomar todas las medidas para salvaguardar la integridad de la tripulación, así también los encargados de la economía nacional en el contexto financiero internacional turbulento deben tomar todas las medidas para asegurar el crecimiento de la actividad económica.

La pregunta básica que anima a este trabajo nuevamente aparece con propiedad: ¿cuál es la función o lugar de una metáfora conceptual en un argumento real? Se puede comenzar a responder esta pregunta, una vez visto el funcionamiento metafórico de las expresiones en este caso, de la siguiente forma de acuerdo con el modelo de análisis de argumentos de Toulmin: 


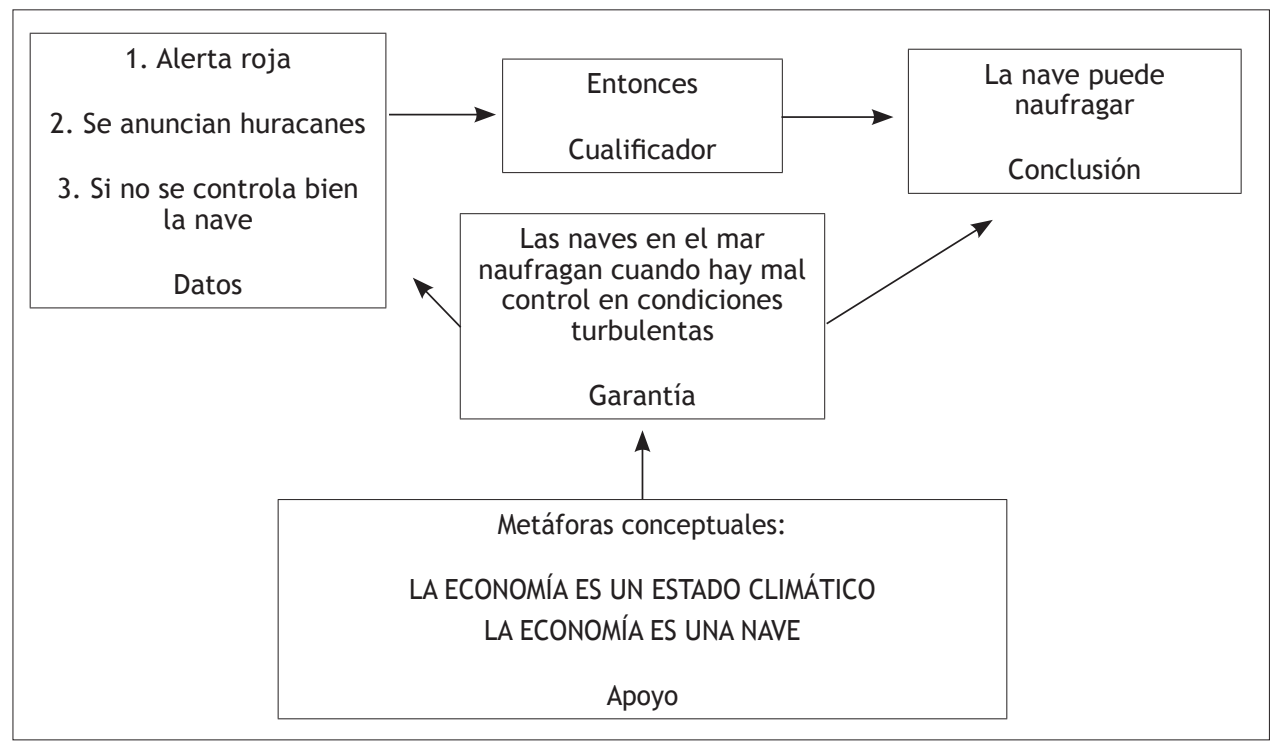

Figura 2. Argumento en términos de dominio fuente.

A través de un proceso de correspondencia, y paráfrasis se debe añadir, entre los dominios conceptuales en el marco de una estructura de inferencia, el argumento en términos de dominio meta se puede representar de la siguiente forma: 


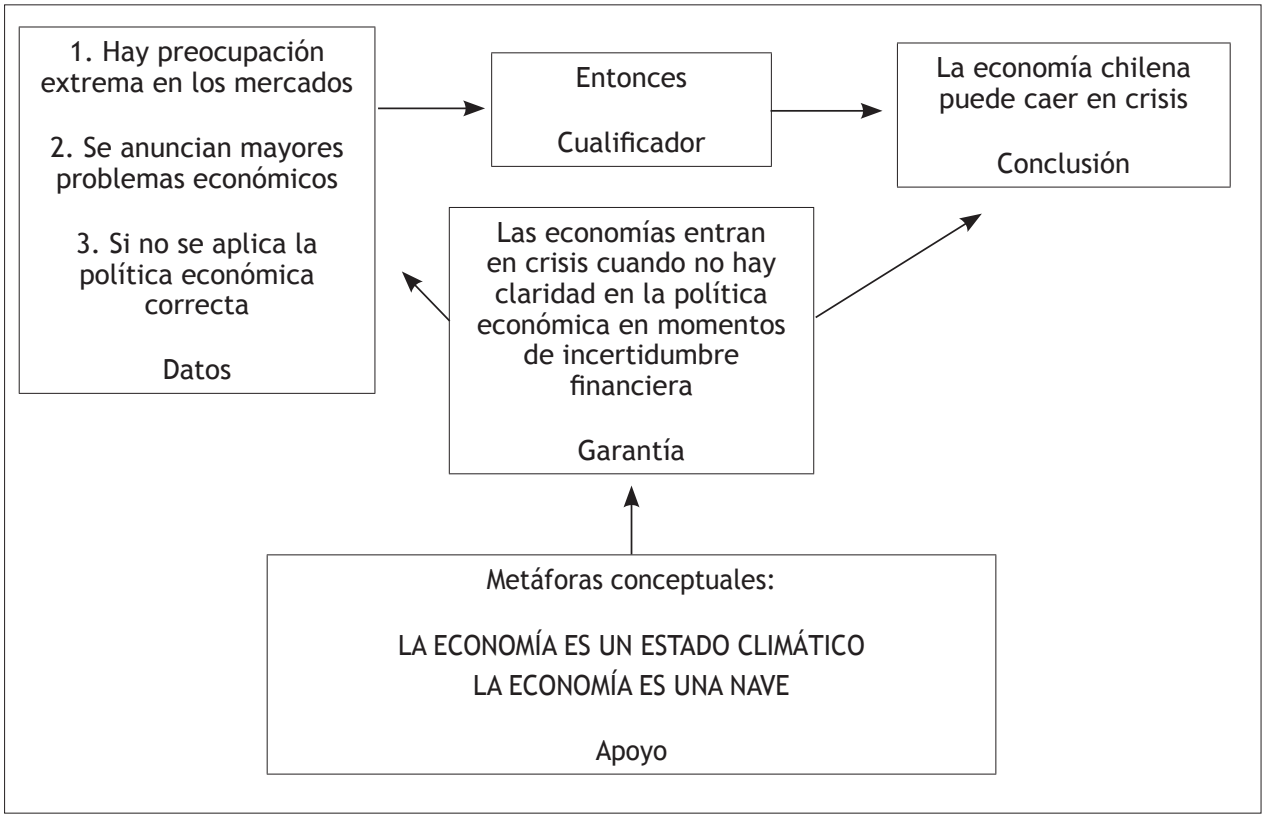

Figura 3. Argumento en términos de dominio meta.

Para observar con toda claridad cómo se despliega la metáfora conceptual como apoyo entre ambos dominio, en la siguiente estructura argumentativa se superponen las correspondencias que contienen la información exportada desde el dominio fuente al dominio meta: 


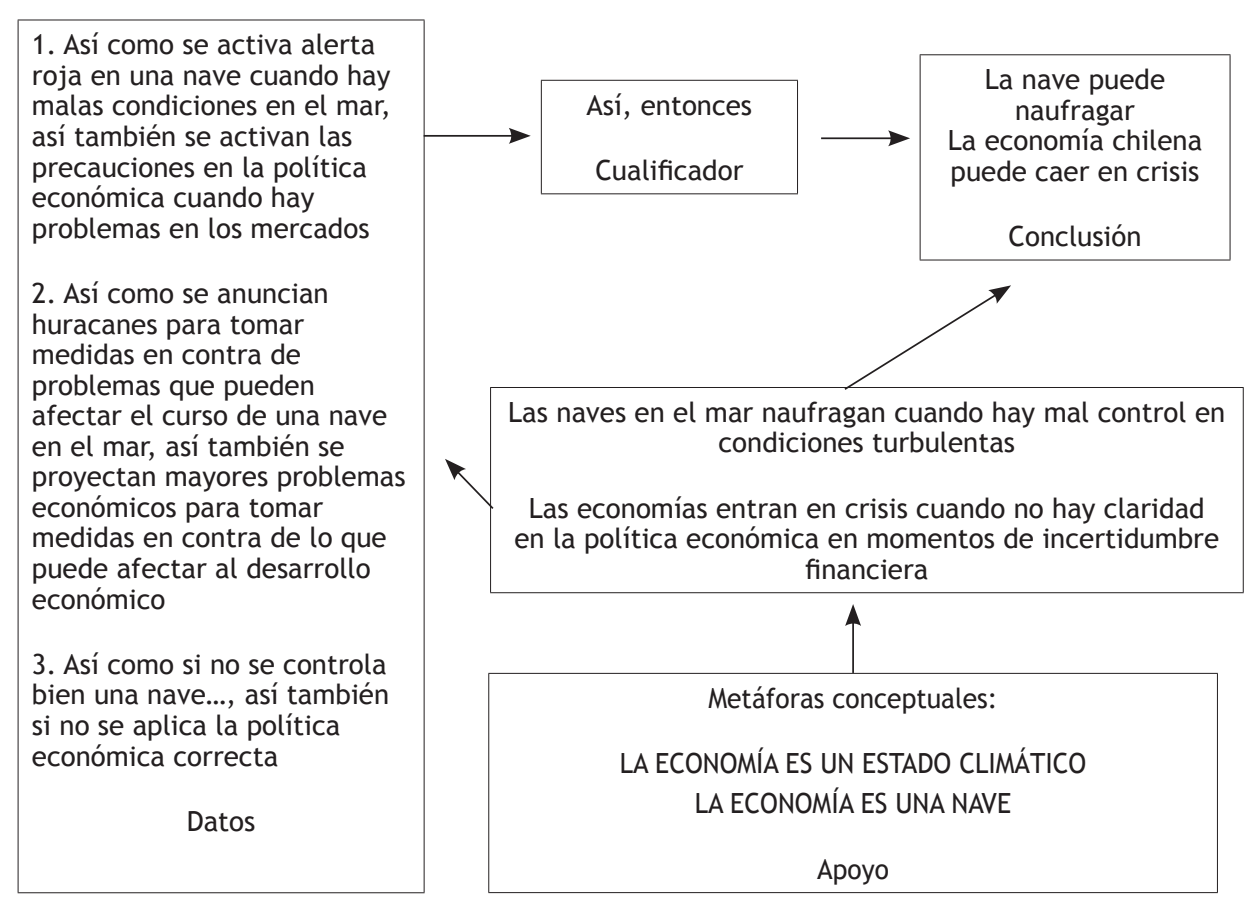

Figura 4. Argumento a partir de correspondencias entre dominios conceptuales.

Las metáforas conceptuales han sido ubicadas en el lugar de los 'apoyos' ya que, como ha propuesto la lingüística cognitiva, funcionan como marcos cognitivos que producen el entendimiento y la acción en el mundo a partir de la experiencia, esto es, nacen como producto de la experiencia que genera, en el transcurso del tiempo, sentido y comprensión de lo que rodea. Para Toulmin et al. (1979: 57), los apoyos son "generalizations making explicit the body of experience relied on to establish the trustworthiness of the ways of arguing applied in any particular case", definición que, justamente, permite realizar el paralelo con cierta seguridad.

Por otra parte, y siguiendo a Lakoff y Johnson (1980), estas dos metáforas conceptuales pueden ser vistas como 'metáforas convencionalizadas', y estos tipos de "metaphors are deeply entrenched and hence well know and widely used in a speech community" (Kövecses, 2002: 248). De acuerdo a Fillmore (1982) y Lakoff (1994), dado cierto marco conceptual, el mecanismo 
semántico generativo de importar-exportar significado entre los dominios marcará siempre la comprensión del concepto o los conceptos involucrados, tal como muestra el ejemplo que se ha analizado. Desde una perspectiva retórica, el hablante podrá enfatizar ciertos aspectos que el marco conceptual contiene, y sus preferencias podrían proyectar los tipos recurrentes de apoyos-marcos sociales respecto de los que el hablante es parte.

\section{2. Metáforas en la vida social y política}

El segundo caso que se analizará trata de la declaración de un parlamentario opositor al gobierno de la Presidenta Michelle Bachelet. Es un caso interesante porque se utiliza una metáfora conceptual estratégicamente para dar un golpe crítico directo.

Las evaluaciones políticas y sociales del modo en que la Presidenta ejerce su cargo han enfatizado constantemente que ella no posee, o que no aplica apropiadamente, su autoridad presidencial. Es una crítica que cruza al espectro político. Una imagen muy gráfica de esto es la siguiente declaración, obtenida del periódico La Nación en su versión electrónica del día 16 de mayo del 2007:

Según Alberto Cardemil, Chile se está pareciendo a la familia "cuya dueña de casa no manda, no tiene claro qué decir; los niños llegan a cualquier hora, se atrasa el almuerzo, no hay plata para la comida, se extravía el presupuesto en materias de gestión que no estaban consideradas, y el dueño de casa sale a emborracharse, cegando las posibilidades de futuro de la misma familia".

\section{Texto 2}

La metáfora conceptual implicada en este extracto es, claramente, una muy vieja y convencionalizada, esto es, arraigada profundamente en las culturas occidentales, que concibe a un 'país' en términos de una 'familia', y, por las propiedades del dominio fuente, al presidente en términos de un 'padre' o 'madre', según sea el caso. Pero que sea una metáfora convencional no significa, desde una perspectiva normativa (van Eemeren \& Grootendorst, 2004), que esté inmune a un uso falaz.

En términos del modelo de Toulmin, el argumento se puede reconstruir como sigue: 


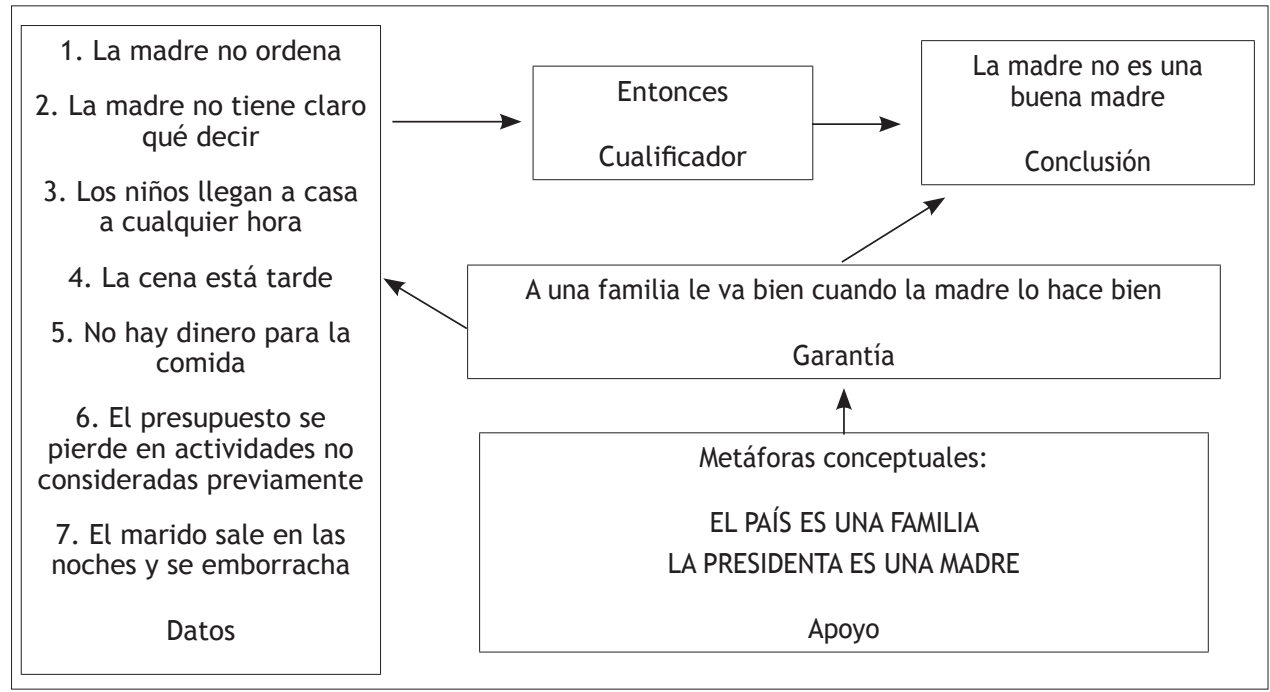

Figura 5. Argumento en términos de dominio fuente.

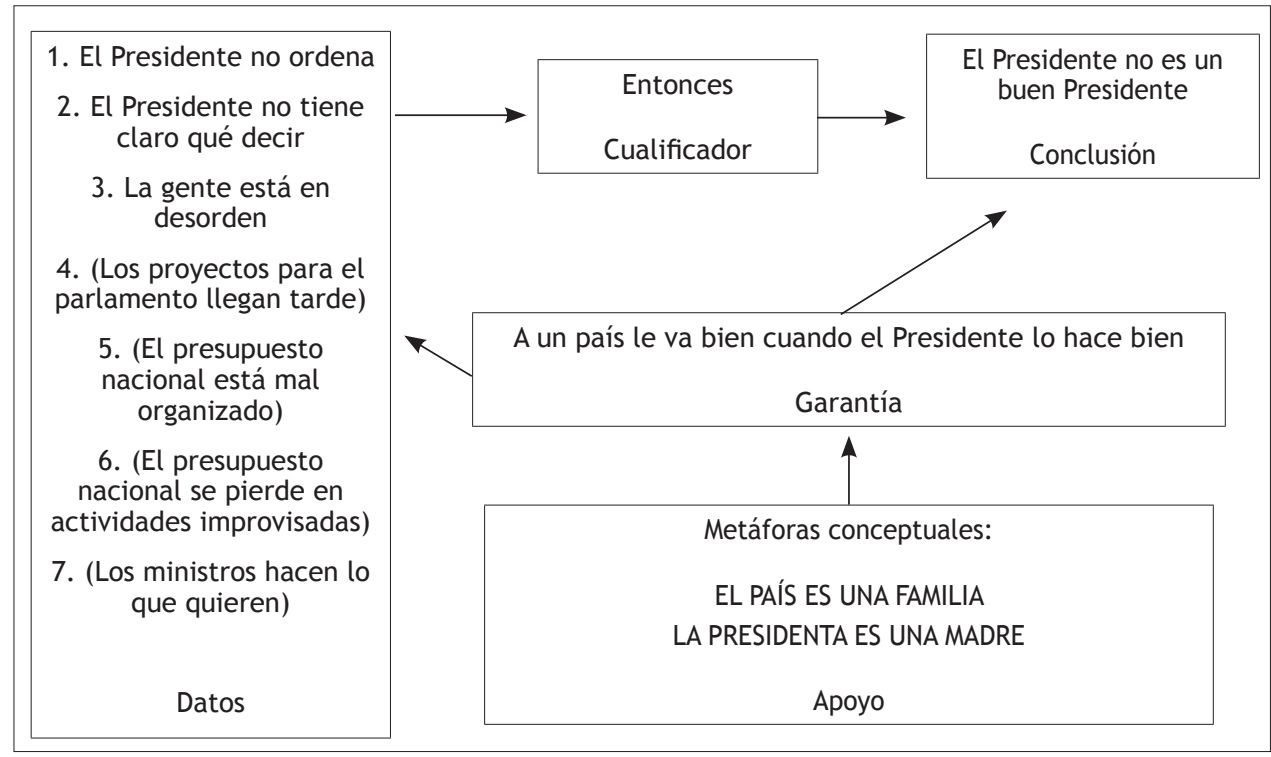

Figura 6. Argumento en términos de dominio meta. 
Han sido puestos entre paréntesis en la reconstrucción argumentativa, en términos de dominio meta, algunos de los datos, ya que no es del todo evidente a qué información del contexto político real se refiere el hablante. Sin embargo, y como se observa, la metáfora es usada para estructurar el punto de vista que la Presidenta no hace un buen trabajo. No obstante, esto se hace sobre la base de una falsa analogía, ya que el esquema argumentativo de comparación, que descansa en el 'principio de justicia', solo puede aplicarse a personas, situaciones o fenómenos que estando en condiciones parecidas pueden ser tratadas de forma similar (Garssen, 2007). Los presidentes de los países no están en condiciones de ser padres o madres de los mismos, por lo que mal se pueden tratar de forma similar. A este tipo de análisis Lakoff (Lakoff, 1992; Chilton \& Lakoff, 1995), como hemos visto, quiere ir acercándose, por lo que una teoría cognitiva de la metáfora se complementa y fortalece con una teoría normativa de la argumentación.

\section{3. Metáforas en las teorías científicas}

No solo en las expresiones cotidianas se ocupan las metáforas. En las ciencias ocurre lo mismo. Incluso las metáforas que vienen incrustadas en los dichos, los proverbios y las expresiones idiomáticas, son utilizadas en este ámbito con fines argumentativos. Estas cristalizaciones del lenguaje han sido estudiadas desde diferentes perspectivas, tanto desde la lingüística cognitiva (Gibbs, 1990; Gibbs \& O’Brien, 1990; Kövecses \& Szabó, 1996; Lakoff \& Turner, 1989), como desde la retórica (Mieder, 2005) y, por cierto, desde el propio punto de vista argumentativo (Goodwin \& Wenzel, 1979; Santibáñez, 2007).

Como el ejemplo del dicho "una manzana podrida echa a perder el cajón", que como vimos Lakoff trae a colación, los enunciados sentenciosos ponen en escena la metáfora conceptual GENÉRICO ES ESPECÍFICO a través de la estructura conceptual esquema-imagen. Al explicar que estas metáforas evocan esquemas ricos en imágenes e información, Lakoff y Turner (1989: 162) señalan que:

"There is a general answer to these questions for all poetry with the characteristics of this proverb [Blind/blames the ditch]. There exists a single generic-level metaphor, GENERIC IS SPECIFIC, which maps a single specific-level schema onto an indefinitely large number of parallel specific-level schemes that all have the same generic-level structure as the source-domain schema".

Este marco general de explicación se aplicará a continuación para observar su productividad analítica. En particular, se utilizará para observar cómo funciona el uso de proverbios, que por naturaleza son metafóricos (Lakoff \& Turner, 1989), en la argumentación científica. En términos del proceso de inferencia, los cuantificadores, los sujetos y los predicados contenidos en un enunciado sentencioso se proyectan, a través del esquema-imagen, a los cuantificadores, sujetos y predicados de la situación específica o contexto en el que se enuncia el proverbio, dicho o 
cliché. El ejemplo que enseguida se expone, y que da cuenta de este proceso, proviene del debate contemporáneo en los estudios de la argumentación en torno al fenómeno de las falacias.

El título del trabajo con el que Jacobs y Jackson (2006) participaron en el tributo a Frans van Eemeren al cumplir éste sus sesenta años, fue: Derailments of argumentation: It takes two to tango -Descarrilamientos en la argumentación: Se necesitan dos para el tango- (Jacobs \& Jackson, 2006: 121). It takes two to tango es, de acuerdo con Mieder (2005: 13), un proverbio norteamericano que ha sido exportado con éxito a otras culturas con el mismo significado y aplicación. Este caso es una muy buena representación del uso de la sabiduría popular en el contexto académico. El esquema que este proverbio contiene, en tanto información de nivel genérico, podría ser caracterizado, al menos, con las siguientes entradas: 1) Hay un baile llamado tango; 2) Este baile no puede ser bailado solo por una persona; 3) Si alguien trata de bailar el tango a solas, no será realmente tango; y 4) Para bailar este baile se necesitan dos personas.

Esta información constituye un esquema genérico que representa al dominio fuente y que puede ser transferido a otros dominios conceptuales para el entendimiento de sus contenidos informativos específicos, y que para nuestro caso viene a ser: 1) Hay un problema en la teoría de la argumentación llamado 'falacia'; 2) Este problema no puede ser explicado por un concepto que considere una sola parte; 3) Si alguien trata de explicar el problema de la 'falacia' con este concepto, no será la explicación correcta; y 4) Para explicar el problema uno necesita considerar las dos partes envueltas en una situación falaz.

Los autores del artículo que incorporan en su título este proverbio no solo utilizan este mecanismo para explicar su posición, sino que también discuten el problema con otros argumentos y ejemplos, y parte medular de sus razones se pueden ver en el siguiente pasaje:

"Whether viewed as a wrongly applied argumentation scheme or as a move that is a functional violation of a discussion rule, we doubt the possibility, and even the desirability, of classifying an individual argument (or other move) as fallacious or not outside of its context of occurrence. Embedding all arguments -strong, weak, and fallacious- in discursive engagements, we are much more interested in the interplay of move and countermove than in extracting one party's contribution alone and inspecting it for fallaciousness. When arguments seem fallacious to a participant, it is that participant's responsibility to initiate repair (or to withhold or adjust those arguments in the first place). Where bad moves may slip by, what is needed are procedures that manage their occurrence in a constructive way... Move and countermove are equally implicated in anything that goes wrong; together they make up flow. Derailment always occurs in at least two steps" (Jacobs \& Jackson, 2006: 124).

En este extracto podemos ver cómo el proverbio impone una línea argumentativa y, al mismo tiempo, cómo las razones específicas están contenidas en el marco que el proverbio provee. 
Colocar el proverbio en el título del artículo no es solo una estrategia retórica básica, sino que también es una clara instrucción argumentativa para entender lo que a juicio de los autores es la correcta lectura del problema que tratan. Es un buen título porque evoca metafóricamente una imagen gráfica a través de la que la crítica que los autores exponen se hace más explícita, confiriéndole al argumento una fuerza particular. Pero, uno debiera ir con cuidado al momento de juzgar si un argumento tiene o no tiene 'fuerza', ya que, como Freeman (2006: 25) señala: "... 'strenght' and 'weight' are obviously metaphorical".

\subsection{Metáforas en la vida parlamentaria}

El caso anterior, que se encuentra en el campo argumentativo 'ciencia' (Toulmin et al., 1979), se repite por cientos en la práctica política cotidiana. El siguiente ejemplo, obtenido del periódico La Tercera de su versión electrónica del 30 de marzo de 2007, así lo refleja:

El senador Demócrata Cristiano Jorge Pizarro sostuvo: "la Presidenta (Bachelet) ya decidió, no hay que darle más vueltas al asunto, hay que arar con los bueyes que se tienen como se dice, y respaldar la gestión de la Presidenta”.

\section{Texto 3}

En este caso, la información genérica que el esquema del proverbio contiene puede ser caracterizada con los siguientes elementos: 1) El agricultor tiene que arar terreno difícil para obtener alimento; 2) El agricultor ara con el mismo buey que siempre ha tenido; 3) El agricultor no puede cambiar fácilmente el buey porque es una gran inversión en términos de dinero y tiempo; 4) Para cambiar el buey el agricultor debe tener una buena justificación; y 5) Para cambiar el buey el agricultor debe tener una alternativa a la mano.

Esta información, que representa al dominio fuente, puede ser transferida al dominio meta en términos de un esquema informativo específico, como a continuación se ensaya: 1) La presidenta tiene que enfrentar problemas difíciles para aplicar su programa político; 2) La presidenta enfrenta su programa con los ministros que tiene; 3 ) La presidenta no puede cambiar fácilmente sus ministros porque es una discusión difícil con los partidos políticos; 4) Para cambiar a los ministros la presidenta debe tener una buena justificación; y 5) Para cambiar los ministros la presidenta debe tener las alternativas a la mano.

Nuevamente, como en el caso anterior, la metáfora implicada en este proverbio permite clarificar una opinión, estructurar un argumento, describir una situación. En este uso, se busca precisar la interpretación que se puede hacer de los sujetos contenidos en la comparación. Así, lo que se enfatiza es la 'resignación' que se debe adoptar para entender que los ministros no se 
pueden cambiar tan fácilmente, tal como un agricultor o un campesino no puede cambiar de la noche a la mañana sus animales de arado. De modo que aquí la comparación no está hecha en virtud de la conceptualización de los 'animales', sino en la escena básica y completa que tiene el uso de determinados animales en determinadas funciones.

\section{CONCLUSIONES}

El esfuerzo por vincular el estudio de la metáfora en términos cognitivos con los estudios teóricos de la argumentación, está en línea con otros esfuerzos generales por balancear la relación entre el estudio de las estrategias retóricas, las estructuras cognitivas y los modelos normativos de análisis de la argumentación (Ponterotto, 2000; Tindale 1992, 1999; van Eemeren \& Houtlosser, 1999, 2002a, 2002b, 2002c), que buscan describir y explicar las formas a través de las que los puntos de vista en discusiones, debates, polémicas o controversias toman expresión. La historia del estudio de la metáfora demanda un intento serio de asumir el desafío de explorar el lugar de la metáfora en las construcciones silogísticas o entimemáticas, ya que no se puede continuar entendiéndola solo en términos retóricos o filosóficos (Aristóteles, 2001; Richards, 1936; Ricoeur, 1977; Searle, 1979), especialmente dada la evidencia general desde un punto de vista cognitivo.

Sabemos que las metáforas son parte de un marco conceptual amplio, tal como los argumentos y la actividad misma de la argumentación son parte de un continuo -polifónico (Ducrot \& Anscombre, 1983)- de diferencias de opinión; pero observadas las metáforas como metáforas conceptuales en un engranaje argumental, ellas pueden ser analizadas como material general o evidencia social para apoyar argumentos, esto es, como 'respaldos', o pueden ser vistas como 'garantías' cuando una de sus correspondencias nos permite ver el movimiento que va desde una razón particular a una conclusión. En el caso del uso de proverbios, la metáfora se convierte en un esquema de inferencia paralelo para enfatizar la ruta que sigue un argumento específico. Mayores análisis permitirán rechazar o refinar estas pretensiones teóricas. Este es un paso.

Un tema que puede ser parte de futuras investigaciones, en una línea de trabajo similar a la que aquí se propuso, sería continuar el estudio de casos reales en los que se utilizan metáforas conceptuales con fines argumentativos para distinguir tipos de hablantes, grupos o comunidades discursivas, de acuerdo con tendencias o recurrencias en el uso de metáforas conceptuales.

Sin embargo, este acercamiento no está exento de problemas o puntos críticos. Uno de ellos dice relación con el problema metodológico de su aplicación, vinculado a la subjetividad del analista que puede escoger aquellos casos en los que el modelo se aplica con facilidad, allí donde, dado un conjunto de expresiones, se puede parafrasear sus correspondencias metafóricas sin mayor obstáculo. Un segundo e importante problema, muy vinculado al anterior, es el de la 
arbitrariedad a la hora de escoger los dominios que implicaría una metáfora conceptual, siendo el nombre mismo de la metáfora conceptual un aspecto crítico. Por ejemplo, al escuchar una expresión como "ella explotó de ira", ¿deberíamos escoger para el dominio fuente un referente o concepto líquido o sólido -LAS PERSONAS SON DINAMITA o LAS PERSONAS SON GASOLINA? Una respuesta parcial a ambos problemas es sostener que hasta donde la reconstrucción o paráfrasis de las expresiones metafóricas y la consecuente obtención de los dominios y la metáfora conceptual misma sean razonables, es decir, sean distinguibles por hablantes ordinarios, las paráfrasis no corren peligro de solipsismo reconstructivo o interpretativo, puesto que toda reconstrucción analítica, en el marco de una teoría de la argumentación, estará en función de un objetivo mayor que podría ser el de entender, describir y analizar una línea argumental o una estrategia retórica. La inteligibilidad cotidiana aun es buen parámetro para discernir métodos en las ciencias humanas.

Un tercer problema es el de la relación entre metáfora y analogía. Desde una perspectiva crítica al análisis metafórico cognitivista, se podría sostener que en este análisis todo es asunto de analogía, que el problema de la comparación es un problema de equilibrio o armonía en la proporción entre los términos o conceptos comparados, por lo que en el análisis metafórico cognitivista no se encuentra más de lo que el procedimiento por analogía permite. Frente a este cuestionamiento, se podría señalar que la analogía es un término vago, tan vago como el de metáfora si se quiere, pero a diferencia de éste, el procedimiento analógico no presenta pasos metodológicos específicos para una reconstrucción acabada, como sí lo hace el análisis metafórico de perfil cognitivista. Por lo demás, Aristóteles (2003) señaló que una cuarta forma o dimensión de la metáfora es su trabajo analógico. Dicho esto, se podría hablar, entonces, de analogía metafórica, siendo la analogía un procedimiento contemplado por la metáfora, que es más amplia.

Un último aspecto problemático de este acercamiento, que aquí solo se puede mostrar, es que el análisis metafórico, así como lo presenta Lakoff y su equipo, se mueve principalmente en terreno de lo descriptivo, siendo el caso que en la teoría argumentativa la mayor parte del trabajo teórico contemporáneo está vinculado al tópico de la evaluación de argumentos. Dicho de otro modo, hablar de una teoría de la argumentación es hablar, ipso facto, de evaluación o normatividad (Johnson, 2000; Johnson \& Blair, 2006). Así, si el análisis metafórico cognitivista quiere tener éxito desde una perspectiva de la argumentación, debe ser incorporado a una teoría que conciba el uso de metáforas como procedimientos sujetos a evaluación crítica, distinguiendo, cuando sea oportuno, cuándo y por qué el uso de una metáfora conceptual deviene en falacia.

Todos estos puntos críticos debieran ser incorporados a una agenda que procure precisar el rol argumentativo de la metáfora conceptual. Por ahora, las preguntas que suscita y las respuestas parciales y alternativas son bienvenidas como parte de algunos elementos de partida. 


\section{REFERENCIAS BIBLIOGRÁFICAS}

Aristóteles. (2001). Retórica. Introducción, traducción y notas de Alberto Bernabé. Madrid: Alianza.

Aristóteles. (2003). Arte poética. Edición bilingüe de Aníbal González. En Aristóteles y Horacio. Artes Poéticas (pp. 46-149). Madrid: Visor.

Chilton, P. \& Lakoff, G. (1995). Foreign policy by metaphor. En Ch. Schaffner \& A. Wenden (Eds.), Languages and peace (pp. 37-59). Brookfield, VT: Dartmouth Publishing Company.

Davidson, D. (1978). What metaphors mean? Critical Inquiry, 5(1) 31-47.

Ducrot, O. \& Anscombre, J. (1983). L'argumentation dans la langue. Belgique: Pierre Mardaga éditeur.

Fillmore, Ch. (1982). Frame semantics. En Linguistic Society of Korea (Ed.), Linguistics in the morning calm (pp. 111-138). Seoul: Hanshin.

Freeman, J. (2006). Argument strength, the Toulmin Model, and ampliative probability. Informal Logic, 26(1) 25-40.

Garssen, B. (2007). Esquemas argumentativos. En R. Marafioti (Ed.), Parlamentos. Teoría de la argumentación y debate parlamentario (pp. 19-34). Buenos Aires: Biblos.

Gibbs, R. (1990). Psycholinguistic studies on the conceptual basis of idiomaticity. Cognitive Linguistics, 1, 417-451.

Gibbs, R. \& O’Brien, J. (1990). Idioms and mental imagery: The metaphorical motivation for idiomatic meaning. Cognition, 36, 35-68.

Goodwin, P. \& Wenzel, J. (1979). Proverbs and practical reasoning: A study in socio-Logic. The Quarterly Journal of Speech, 65, 289-302.

Jacobs, S. \& Jackson, S. (2006). Derailments of argumentation: It takes two to tango. En P. Houtlosser \& A. van Rees (Eds.), Considering pragma-dialectics (pp. 121-134). Mahwah, N.J.: Erlbaum.

Johnson, R. (2000). Manifest rationality. A pragmatic theory of argument. Hillsdale, N.J.: Erlbaum.

Johnson, R. \& Blair, T. (2006). Logical self-defense. New York: International Debate Education Association.

Kövecses, Z. (2002). Metaphor. A practical introduction. Oxford and New York: Oxford University Press.

Kövecses, Z. \& Szabó, P. (1996). Idioms: A view from cognitive linguistics. Applied Linguistics, 17(3), 326-355.

Lakoff, G. (1992). Metaphors and war: The metaphor system used to justify war in the Gulf. En M. Putz (Ed.), Thirty years of linguistics evolution (pp. 463-481). Amsterdam: Benjamins.

Lakoff, G. (1994). What is conceptual system? En W. Overton \& D. Palermo (Eds.), The nature and ontogenesis of meaning (pp. 41-90). Hillsdale, N. J.: Erlbaum.

Lakoff, G. (2004). Don't think of an elephant. Know your values and frame the debate. Vermont: Chelsea Green Publishing. 
Lakoff, G. (2006a). Thinking points. Communicating our American values and vision. New York: Farrar, Straus Giroux.

Lakoff, G. (2006b). Whose freedom? The battle over America's most important idea. New York: Farrar, Straus and Giroux.

Lakoff, G. \& Johnson, M. (1980). Metaphors we live by. Chicago: University of Chicago Press.

Lakoff, G. \& Turner, M. (1989). More than cool reason. Chicago: University of Chicago Press.

Lanham, R. (1991). A handlist of rhetorical terms. Berkeley: University of California Press.

Mieder, W. (2005). Proverbs are the best policy. Folk wisdom and American politics. Logan, Utah: Utah State University Press.

Perelman, Ch. \& Olbrechts-Tyteca, L. (2000). The new rhetoric. A treatise on argumentation. Notre Dame: University of Notre Dame Press.

Ponterotto, D. (2000). The cohesive role of cognitive metaphor in discourse and conversation. En A. Barcelona (Ed.), Metaphor and metonymy at the crossroads (pp. 283-298). Berlin: Gruyter.

Richards, I. (1936). The Philosophy of rhetoric. New York: Oxford University Press.

Ricoeur, P. (1977). The rule of metaphor: Multidisciplinary studies of the creation of meaning in language. Toronto: University of Toronto Press.

Santibáñez, C. (2007). Sayings in political discourse: Argumentative and rhetorical uses. En F. van Eemeren, A. Blair, Ch. Willard \& B. Garssen (Eds.), Proceedings of the Sixth Conference of the International Society for the Study of Argumentation (pp. 1227-1232). Amsterdam: Sic Sat.

Searle, J. (1979). Expression and meaning. Cambridge: Cambridge University Press.

Tindale, C. (1992). Audiences, relevance, and cognitive environnements. Argumentation, 6, 177188.

Tindale, C. (1999). Acts of arguing. A rhetorical model of argument. Albany: State University of New York Press.

Toulmin, S. (1958). The uses of argument. Cambridge: Cambridge University Press.

Toulmin, S., Rieke, R. \& Janik, A. (1979). An introduction to reasoning. New York: Macmillan Publishing Co., Inc.

Van Eemeren, F. \& Grootendorst, R. (1992). Argumentation, communication, and fallacies. A pragma-dialectical perspective. Hillsdale, N.J.: Erlbaum.

Van Eemeren, F. \& Grootendorst, R. (2004). A systematic Theory of Argumentation. The pragmadialectical approach. Cambridge: Cambridge University Press.

Van Eemeren, F. \& Houtlosser, P. (1999). Strategic maneuvering in argumentative discourse. Discourse Studies, 1, 479-497.

Van Eemeren, F. \& Houtlosser, P. (2002a). Strategic maneuvering with the burden of proof. En F. van Eemeren (Ed.), Advances in pragma-dialectics (pp. 13-28). Amsterdam: Sic Sat. 
Van Eemeren, F. \& Houtlosser, P. (2002b). Fallacies as derailments of strategic maneuvering: The argumentum ad verecundiam, a case in point. En F. van Eemeren, A. Blair, Ch. Willard \& F. Snoeck (Eds.), Proceedings of The Fifth Conference of The International Society for The Study of Argumentation (pp. 289-292). Amsterdam: Sic Sat.

Van Eemeren, F. \& Houtlosser, P. (2002c). Strategic maneuvering: Maintaining a delicate balance. En F. van Eemeren \& P. Houtlosser (Eds.), Dialectic and rhetoric: The warp and woof of argumentation analysis (pp. 131-159). Dordrecht: Kluwer.

Van Eemeren, F. \& Houtlosser, P. (2003). Fallacies as derailments of strategic maneuvering: The argumentum ad verecundiam, a case in point. En F. van Eemeren, J. Blair, C. Willard \& A. Snoeck (Eds.), Proceedings of the Fifth Conference of the International Society for the Study of Argumentation (pp. 289-292). Amsterdam: Sic Sat.

Van Eemeren, F. \& Houtlosser, P. (2008). Strategic manoeuvring in argumentative discourse: Exploring the boundaries of reasonable discussion. En F. van Eemeren, D. Cratis \& I. Zagar (Eds.), Understanding Argumentation (pp. 13-26). Amsterdam: Sic Sat-Rozenberg.

Walton, D. (1992). Plausible argument in everyday conversation. Albany: State University of New York Press.

Walton, D. (2004). Abductive reasoning. Tuscaloosa: The University of Alabama Press.

Walton. D. (2006). Fundamentals of critical argumentation. Cambridge: Cambridge University Press.

Walton, D. (2007). Media argumentation. Dialectic, persuasion, and rhetoric. Cambridge: Cambridge University Press. 